\title{
Information-Based Hierarchical Planning for a Mobile Sensing Network in Environmental Mapping
}

\author{
Teng $\mathrm{Li}^{\circledR}$, Member, IEEE, Kaitai Tong ${ }^{(\circledR)}$, Min Xia ${ }^{\circledR}$, Member, IEEE, Bing $\mathrm{Li}^{\circledR}$, and \\ Clarence Wilfred de Silva ${ }^{\circledR}$, Fellow, IEEE
}

\begin{abstract}
This article investigates the problem of informationbased sampling design and path planning for a mobile sensing network to predict scalar fields of monitored environments. A hierarchical framework with a built-in Gaussian Markov random field model is proposed to provide adaptive sampling for efficient field reconstruction. In the proposed framework, a nonmyopic planner is operated at a sink to navigate the mobile sensing agents in the field to the sites that are most informative. Meanwhile, a myopic planner is carried out on board each agent. A tradeoff between computationally intensive global optimization and efficient local greedy search is incorporated into the system. The mobile sensing agents can be scheduled online through an anytime algorithm to visit and observe the high-information sites. Experiments on both synthetic and real-world datasets are used to demonstrate the feasibility and efficiency of the proposed planner in model exploitation and adaptive sampling for environmental field mapping.
\end{abstract}

Index Terms - Adaptive sampling, environmental field mapping, Gaussian Markov random fields (GMRFs), information-driven planning, mobile sensing networks (MSNs).

\section{INTRODUCTION}

$\mathbf{M}$ OBILE sensing networks (MSNs) can provide unprecedented flexibility, efficiency, and effectiveness in information gathering. They can improve the performance of an environmental monitoring process. With the support of mobile sensing, measurements of the monitored variables over both spatial and temporal scales can be collected to estimate, interpret, and reconstruct the environmental field of interest. Due to these advantages, MSNs have been developed and deployed to provide in situ measurements and field maps in many environmental monitoring programs [1]-[3].

When implementing an MSN, owing to the limited number and mobility of the sensing agents (e.g., mobile sensors or

T. Li and C. W. de Silva are with the Department of Mechanical Engineering, University of British Columbia, Vancouver, BC V6T 1Z4, Canada (e-mail: tengli@mech.ubc.ca; desilva@mech.ubc.ca).

K. Tong is with the Faculty of Applied Science, University of British Columbia, Vancouver, BC V6T 1Z4, Canada (e-mail: kaitai@alumni.ubc.ca).

M. Xia is with the Department of Engineering, Lancaster University LA1 4YW Lancaster, U.K. (e-mail: m.xia3@lancaster.ac.uk).

B. Li is with the Harbin Institute of Technology Shenzhen Graduate School, Shenzhen 518055, China (e-mail: bingli@ hit.edu.cn). vehicles), the sites that can be visited within any period of time is bounded. Furthermore, the resource constraints of the system (e.g., power supply [4]) impede its operating life. Quite importantly, redundant data collections will not lead to information gain but will use up the system energy and can also exceed the onboard computational capacity. Therefore, the agents should take samples at more representative and high-information sites, to capture and predict the underlying environmental variation more efficiently and effectively.

The selection of the sampling sites that are more crucial in making predictions of the spatial field is an experimental design problem [5]. The design techniques determine the target sites for data collection by exploiting a statistical model of the monitored environment. Studies on information-theoretic sensing have indicated their power on exploiting the sites that are most informative in a Gaussian random field (GRF) through a Gaussian process (GP) model [6]. The optimized design of a GP model has been proven nondeterministic polynomial-time (NP)-hard [7]. The NP-hard characteristic leads to high computational complexity as the number of measurements grows. To promote the computational efficiency, many statistical schemes have been introduced to approximate the GP model [8], [9]. Although reducing the algorithm complexity of the GPs, these schemes are still computationally intractable, especially for a large-scale area.

In MSN-based applications, it is crucial for mobile sensing agents to provide the capabilities of online sampling, autonomous navigation, and real-time decision-making. Therefore, the execution process of sampling design and path planning is required in real time or at least near real time. However, the NP-hard characteristic of the GP scheme makes it challenging to achieve online implementation of information-theoretic sensing. The optimization of a sampling plan in the GP dynamics is impractical for mobile sensing scenarios, even when a greedy solution of a discretized space is utilized [10]. This article motivates the handling of online planning of information-driven mobile sensing for environmental field mapping. It focuses on contributing to carry out information-theoretic optimization of a GRF in an efficient and reliable manner.

The present article proposes a hierarchical planner for an MSN to gather information adaptively and efficiently for environmental field mapping. The proposed planner provides a feasible framework for bridging the gap between global informationtheoretic optimization and shortsighted local greedy planning. A Gaussian Markov random field (GMRF) model is built in the proposed framework to represent the underlying field, which 
further determines the information quantities of the sampling locations. The main structures and contributions of the proposed algorithm are as follows.

1) The high-level planner designs the target sites that are most informative in a global and nonmyopic view over the entire spatial field, which is executed as an anytime algorithm at the sink to navigate the mobile sensors continuously over time.

2) The low-level planner searches the high-information locations locally and myopically while heading to the optimal sites that are assigned by the higher level planner, which ensures real-time planning of the mobile sensors.

3) The proposed hierarchical planning framework provides an online and anytime planning solution by integrating both computationally intensive global optimization and fast local greedy search, which addresses the effect of sequential sampling in the field on the future global optimization at the sink.

The remainder of this article is organized as follows. Section II introduces the related work in the field of mobile sensing, experimental design, and information-based planning. The preliminaries and the problem formulation in this article are provided in Sections III and IV, respectively. In Section V, the proposed hierarchical planning framework is presented in detail. Experimental results using both synthetic and real-world datasets are presented and discussed in Section VI. The final section concludes the article.

\section{RELATED WORK}

GP, a nonparametric Bayesian scheme, has been actively studied to model underlying environments and to provide estimation, interpretation, and prediction of the study area [11]. Conditioned on the measurements, inferring the posterior variable distribution (mean and variance) of the GRF results in the field scalar map and the prediction uncertainty, which is known as Gaussian process regression (GPR) or Kriging [12]. However, the GP class is computationally intractable since the computation dependence is cubic with respect to its covariance function when factorizing it in making predictions. For more efficient processing, the GMRF approaches have been introduced to approximate the GPs by designating the Markovian property into the GRFs [13]. In this manner, the computation scale mainly depends on sparse precision matrix, which effectively reduces the computational complexity.

For an unknown field, in a pilot study or a prior survey, exploratory sampling has been studied to observe and learn the statistical model of the underlying field. A set of model parameters, termed as hyperparameters, is used to represent the specific model of a stochastic phenomenon. In the literature, many exploration strategies for mobile agents in a sensing network have been suggested to estimate uncertain hyperparameters. For example, Xu et al. proposed a GP scheme with a built-in GMRF [14] to represent a wide range of physical processes and update predictive statistics through a sequential field prediction algorithm. Then, Xu et al. [15] developed a Bayesian spatial predictor to infer the uncertain GMRF hyperparameters through myopic adaptive sensing. By considering future observations, Nguyen et al. [16] presented an information-driven adaptive sampling planner for GP learning and regression. Recently, the work in [17] further investigated field exploration by incorporating path integral control and statistical learning with GMRFs.
Another class of research investigates information-based sensing of a random field using its statistical model structure. With more a priori knowledge and less uncertainty about the hyperparameters (through exploration), exploiting the statistical model can select more crucial sampling sites for field mapping. An optimal design can determine a well-performed and energy-efficient sampling frame, which benefits mobile sensing in persistent monitoring. In the literature, experimental design theory has been studied for the development of optimality criteria, primarily focusing on optimizing an information gain that is determined by an established statistical model. "Alphabetical" optimality approaches have been studied extensively relying on the properties of the corresponding information matrices [18]. In addition, conditional entropy (CE) and mutual information (MI) have been adopted as information-theoretic metrics to evaluate the high-information sites [19], [20], which quantify the information gain of a sampling frame in terms of prediction uncertainty.

In the fields of mobile sensor networks and robotics, information-based planning tackles the problem of generating a sampling schedule or trajectory that maximizes the information gain through the planned mission. The present article primarily focuses on online planning of an information-driven experimental design for MSN-based environmental mapping, by exploiting the spatial statistical model of the monitored field. With a similar research motivation, nonmyopic informationbased planning has been investigated to optimize a sampling frame by integrating the global information over the study area [21]. In a recent work, Evans et al. [22] planned informative paths by leveraging a priori knowledge about the GP model of the spatial field. Ma et al. [23] proposed an information-based planner for a single robot, which generated the waypoints that were maximally informative based on the MI criterion.

Maximizing the information-theoretic utility functions in the GRFs is proven to be NP-hard and impractical even for discrete cases [24]. Nearly optimal greedy solutions have been proposed for variants of GP dynamics (e.g., [6], [25]), and still, the required computational effort is intractable for a large-scale problem. Also, despite the optimization complexity, many efforts consider the sampling design and the path planning as two consequential procedures. These planners connect the designed target sites through a graph-based method [23], [26], which also suffer from NP-hard complexity. Furthermore, most nonmyopic approaches calculate the maximally informative sites at once without considering the data samples from sequential sampling. When designing a set of target sites for sequential sampling, the effect of future data samples while traveling towards them should be taken into account. To tackle these problems, the present article develops information-based planning for MSNbased environmental field mapping. In particular, the proposed hierarchical planner provides an effective and efficient solution for online planning by integrating nonmyopic and myopic search in the sensing system.

\section{PRELIMINARIES}

Some general notations and concepts are presented here. $\mathbb{R}$, $\mathbb{Z}$, and $\mathbb{Z}^{+}$denote the set of reals, integers, and positive integers, respectively. $\operatorname{Exp}(\cdot), \operatorname{Var}(\cdot)$, and $\operatorname{Cov}(\cdot)$ denote the functions of probabilistic expectation, variance, and covariance of random variables, respectively. $\|\cdot\|$ denotes the length of a Euclidean vector and $|\cdot|$ denotes the determinant of a matrix. 


\section{A. Spatial Field Model in GMRF}

In the present article, a spatial field is considered as a twodimensional plane $\mathcal{A} \subset \mathbb{R}^{2}$. The field is discretized into a set of sampling locations $\mathcal{S}=\left\{\mathbf{s}_{1}, \mathbf{s}_{2}, \ldots, \mathbf{s}_{N}\right\}$. Each location is indexed by $n \in \mathcal{N}:=\{1,2, \ldots, N\}$ and $\mathbf{s}_{n}=\left(s_{1}, s_{2}\right) \in \mathbb{R}^{2}$. In the context of GP, a GRF is defined by any finite collection of random variables at their corresponding locations as

$$
\mathbf{Z}_{\mathcal{S}}=\left(Z\left(\mathbf{s}_{1}\right), Z\left(\mathbf{s}_{2}\right), \ldots, Z\left(\mathbf{s}_{N}\right)\right)^{T} \sim \mathcal{G} \mathcal{P}(\boldsymbol{\mu}, \boldsymbol{\Sigma})
$$

where the process $\mathbf{Z}_{\mathcal{S}}$ is specified by the mean function $\boldsymbol{\mu} \in \mathbb{R}^{N}$ and the covariance function $\boldsymbol{\Sigma} \in \mathbb{R}^{N \times N}$. The elements of the mean function are the expectations of the random variables. The elements of the covariance function give the variable covariance, i.e., $\Sigma_{i j}=\operatorname{Cov}\left(\mathbf{s}_{i}, \mathbf{s}_{j}\right), i \neq j \in \mathcal{N}$. A covariance function is symmetric positive-definite and describes the spatial correlation between different random variables. Matérn class of covariance functions has been widely studied in GP modeling [27], which can be expressed as

$$
\boldsymbol{\Sigma}\left(\mathbf{s}_{i}, \mathbf{s}_{j}\right)=\frac{\sigma^{2}}{\Gamma(\nu) 2^{\nu-1}}(\kappa h)^{\nu} K_{\nu}(\kappa h)
$$

where $h=\left\|\mathbf{s}_{i}-\mathbf{s}_{j}\right\|$ denotes the Euclidean distance between $\mathbf{s}_{i}$ and $\mathbf{s}_{j}, \sigma^{2} \in \mathbb{R}$ denotes the marginal variance, $\Gamma$ denotes the Gamma function, $\nu$ denotes the Matérn smoothness, $\kappa$ denotes the spatial scale parameter, and $K_{\nu}(\cdot)$ denotes the modified Bessel function. The Matérn class is a generalization of the radial basis function with additional parameters to smooth the estimated covariance function. It can yield realistic results to match physical processes due to its finite differentiability with respect to finite smoothness [27].

For notation simplicity, the GRF $\mathbf{Z}_{\mathcal{S}}$ is denoted as $\mathbf{Z}=\left(Z_{1}, Z_{2}, \ldots, Z_{N}\right)^{T}$. Let $\mathbf{Z}_{-i}$ denote the GRF without the random variable $Z_{i}, i \in \mathcal{N}$, and $N E_{i}$ denote the index set of neighbors to a sampling location $\mathbf{s}_{i} \in \mathcal{S}$. If the neighbors in $N E_{i}$ satisfy that $p\left(Z_{i} \mid \mathbf{Z}_{-i}\right)=p\left(Z_{i} \mid \mathbf{Z}_{N E_{i}}\right)$, the spatial field follows the Markovian property and can be modeled as a GMRF. The GMRF is specified as

$$
\mathbf{Z} \sim \mathcal{G} \mathcal{M R \mathcal { F }}\left(\boldsymbol{\mu}, \mathbf{Q}^{-1}\right)
$$

with the mean function $\boldsymbol{\mu} \in \mathbb{R}^{N}$ and the precision matrix $\mathbf{Q} \in$ $\mathbb{R}^{N \times N}$. The precision matrix is the inverse of the covariance matrix of the GP, i.e., $\mathbf{Q}=\boldsymbol{\Sigma}^{-1}$. The elements in $\mathrm{Q}$ are nonzero only for neighbors and diagonal elements, that is, the precision matrix is sparse. The Matérn kernel can be explicitly solved to obtain the precision matrix $Q$ in the context of GMRF. By using the precision matrix $\mathbf{Q}$, there is no need to factorize the dense covariance function $\boldsymbol{\Sigma}$ when operating model-based information-driven optimization. The sparseness of the precision matrix effectively alleviates the computational complexity. This process can help achieve the research motivation of online planning for mobile sensing.

The work in [13] revealed the explicit connection between the GPs and the GMRFs through the Matérn class. It was proven that, for any triangulation of the field, the linear fractional stochastic partial differential equation (SPDE) derived the precision matrix as the inverse of the Matérn covariance function. To express the GMRF as the solution of SPDE, an unstructured triangulation is required to construct a finite element representation for designating the Markovian property of the study field. A triangular mesh is generally constructed for the studied field to define the vertices and their neighbors. The generated mesh forms an undirected graph $\mathcal{G}=\{\mathcal{V}, \mathcal{E}\}$, where the vertices $\mathbf{v} \in \mathcal{V}$ discretize the continuous domain $\mathcal{A}$ to an indexed GMRF while

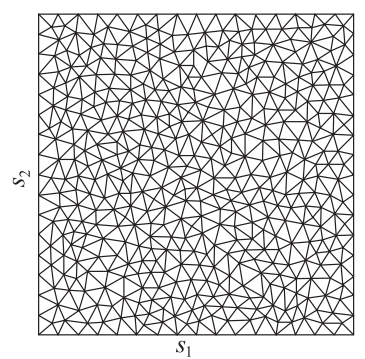

Fig. 1. Triangular mesh of the discretized spatial field $\mathcal{A} \subset \mathbb{R}^{2}$.

the edges $\mathbf{e} \in \mathcal{E}$ indicate the corresponding neighbors. A triangulation example is shown in Fig. 1. The GMRF model is specified by the sparse precision matrix in terms of the constructed graph. The SPDE approach calculates the explicit solution of the elements of $\mathbf{Q}$ corresponding to a Matérn covariance function. For two-dimensional domains, $\mathrm{Q}$ can be obtained as

$$
\mathbf{Q}=\tau^{2}\left(\kappa^{4} \mathbf{C}+2 \kappa^{2} \mathbf{G}+\mathbf{G} \mathbf{C}^{-1} \mathbf{G}\right)
$$

where the elements of $\mathbf{Q}$ are specified by the hyperparameter vector $\boldsymbol{\theta}=(\tau, \kappa)$; the elements of the matrices $\mathbf{C}$ and $\mathbf{G}$ are defined by the inner products of piecewise linear basis functions. More details of (4) are found in [13].

\section{B. Model Learning and Mapping}

By performing a sensing mission, the collected observations $\mathbf{y}$, at their corresponding sampling locations $\mathcal{S}$, can be utilized to learn the hyperparameter vector $\boldsymbol{\theta}$ of the field model. Marginal likelihood maximization with cross validation [28] has been broadly adopted. It estimates the hyperparameter vector by the derivatives of the logarithmic marginal likelihood $\log p(\mathbf{Z} \mid \boldsymbol{\theta})$ with respect to $\theta$ through a gradient-based optimizer, where the log-likelihood of the GMRF model can be obtained as

$$
\log p(\mathbf{Z} \mid \boldsymbol{\theta})=-\frac{N}{2} \log 2 \pi+\log |\mathbf{Q}(\boldsymbol{\theta})|-\frac{1}{2} \mathbf{Z}^{T} \mathbf{Q}(\boldsymbol{\theta}) \mathbf{Z} .
$$

For environmental mapping, the entire field $\mathcal{A}$ is discretized into a grid plane $\mathcal{I}$ where the physical quantities at the grid vertices (query locations) $\mathbf{i} \in \mathcal{I}$ interpret the scalar map. With the sampled data and the field model, the scalar map is inferred by a regression process. In the GMRF/SPDE method, the predictive posterior distribution (mean and variance) conditioned on the samples can be obtained as

$$
\begin{array}{r}
\operatorname{Exp}\left(\mathbf{Z}_{\mathcal{I}} \mid \mathbf{y}\right)=\boldsymbol{\mu}+\mathbf{Q}_{\mathcal{I} \mid \mathcal{S}}^{-1} \mathbf{A}^{T} \mathbf{Q}_{\epsilon}(\mathbf{y}-\mathbf{A} \boldsymbol{\mu}) \\
\operatorname{Var}\left(\mathbf{Z}_{\mathcal{I}} \mid \mathbf{y}\right)=\mathbf{Q}_{\mathcal{I} \mid \mathcal{S}}^{-1}=\left(\mathbf{Q}+\mathbf{A}^{T} \mathbf{Q}_{\epsilon} \mathbf{A}\right)^{-1}
\end{array}
$$

where $\mathbf{A}$ is the projector matrix that maps the mesh vertices to the query locations [29]. Consequently, the field mean $\operatorname{Exp}\left(\mathbf{Z}_{\mathcal{I}} \mid \mathbf{y}\right)$ and the variance $\operatorname{Var}\left(\mathbf{Z}_{\mathcal{I}} \mid \mathbf{y}\right)$ indicate the spatial field map and the estimation uncertainty, respectively.

\section{Informative-Theoretic Metric}

To adaptively sample the data, the sites that are more significant in relation to the information content should be visited. The MI, an information-theoretic metric, is utilized as a utility function to evaluate the information content or the "informativeness" between random variables and find the optimal locations that can maximally reduce the entropy over the unobserved locations. For a random variable $Z_{n}$ at a possible sampling location $\mathbf{s}_{n}$ and the random variables $\mathbf{Z}_{-n}$ at the remaining locations $\mathcal{S} \backslash \mathbf{s}_{n}$, their MI 
is defined as

$$
\operatorname{MI}\left(Z_{n} ; \mathbf{Z}_{-n}\right)=H\left(\mathbf{Z}_{-n}\right)-H\left(Z_{n} \mid \mathbf{Z}_{-n}\right)
$$

where the first and second terms represent the entropy and the conditional entropy of the random variables, respectively. The entropy of a random variable can be calculated by [6]

$$
H(\mathbf{Z})=-\int p(\mathbf{Z}) \log p(\mathbf{Z}) d \mathbf{Z}=\frac{1}{2} \log \left((2 \pi e)^{N}|\mathbf{\Sigma}|\right) .
$$

Since $\mathbf{Q}=\boldsymbol{\Sigma}^{-1}$, the general entropy formulation in (9) can be obtained as

$$
H(\mathbf{Z})=-\frac{1}{2} \log \left((2 \pi e)^{N}|\mathbf{Q}|\right) .
$$

Thus, the entropy $H\left(\mathbf{Z}_{-n}\right)$ and the conditional entropy $H\left(Z_{n} \mid \mathbf{Z}_{-n}\right)$ can be derived, respectively, as

$$
\begin{aligned}
H\left(\mathbf{Z}_{-n}\right) & =-\frac{1}{2} \log \left((2 \pi e)^{N}\left|\mathbf{Q}_{-n}\right|\right) \\
H\left(Z_{n} \mid \mathbf{Z}_{-n}\right) & =-\frac{1}{2} \log \left((2 \pi e)^{N}\left|\mathbf{Q}_{n \mid-n}\right|\right)
\end{aligned}
$$

where $\mathrm{Q}$ can be obtained by the SPDE solver in (4). Consequently, the MI in (8) is obtained as a closed-form expression for a multivariate GMRF as

$$
\operatorname{MI}\left(Z_{n} ; \mathbf{Z}_{-n}\right)=-\frac{1}{2} \log \left|\mathbf{Q}_{-n}\right|+\frac{1}{2} \log \left|\mathbf{Q}_{n \mid-n}\right|
$$

\section{PROBLEM Formulation}

In the present article, the physical phenomenon of a spatial field of interest can be specified in the GMRF scheme as

$$
\mathbf{Y}=\left(Y_{1}, Y_{2}, \ldots, Y_{N}\right)^{T}=\mathbf{Z}+\boldsymbol{\epsilon}
$$

where $\mathbf{Z}$ is a GMRF with mean $\boldsymbol{\mu}$ and precision matrix $\mathbf{Q}$, $\mathbf{Z} \sim \mathcal{G} \mathcal{M} \mathcal{R} \mathcal{F}\left(\boldsymbol{\mu}, \mathbf{Q}^{-1}\right)$, and $\boldsymbol{\epsilon}$ is an independent and identically distributed (i.i.d) noise process with variance $\sigma_{\epsilon}^{2}$.

For an MSN, let the set $\mathcal{U}=\left\{u_{1}, u_{2}, \ldots, u_{M}\right\}$ represent $M \in \mathbb{Z}^{+}$mobile sensing agents that are indexed by $m \in$ $\{1,2, \ldots, M\}$. Each agent $u_{m}$ follows its planned sampling path $p_{m}=\left(\mathbf{s}_{m, 0}, \mathbf{s}_{m, 1}, \ldots, \mathbf{s}_{m, N_{m}}\right)$ with a sequence of sampling sites $\mathbf{s}_{m, k} \in \mathcal{S}$ that are indexed by $k \in\left\{0,1,2, \ldots, N_{m}\right\}$. Following a sampling path $p_{m}$, the agent $u_{m}$ takes a data sample at its current location $\mathbf{s}_{m, k}$ where a physical quantity is observed from a random variable that is expressed as

$$
Y_{m, k}=Z_{m, k}+\epsilon_{m, k} \text {. }
$$

Let $y_{m, k}$ denote an observation of the random variable $Y_{m, k}$. Let $\mathcal{S}_{0: T}$ and $\mathbf{y}_{0: T}$ denote the set of the sampled locations and the corresponding measurements from time 0 to time $T$, respectively. Let $T_{c}$ denote the time interval of an overall sensing cycle, i.e., $T \leq T_{c}$.

The general idea of the proposed planning framework is to guide each agent to sequentially move toward and sample a group of target destinations that are globally optimized, meanwhile sampling high-information locations locally along the sampling trajectory over the travel duration. The informativeness of the sampling locations is measured by the MI submodular utility function, whose maximization through greedy searching is used to derive the global and local optimization design. Given the sampled data at $\mathcal{S}_{0: T}$, the MI between a possible unsampled site $\mathbf{s}_{n} \in \mathcal{S} \backslash \mathcal{S}_{0: T}$ and the remaining unsampled locations $\overline{\mathcal{S}}:=\mathcal{S} \backslash\left(\mathcal{S}_{0: T} \cup \mathbf{s}_{n}\right)$ indicates the target greedy utility, which can be expressed as

$$
\delta=\operatorname{MI}\left(Y\left(\mathbf{s}_{n}\right) ; \mathbf{Y}_{\overline{\mathcal{S}}}\right)=H\left(\mathbf{Y}_{\overline{\mathcal{S}}}\right)-H\left(\mathbf{Y}_{\overline{\mathcal{S}}} \mid Y\left(\mathbf{s}_{n}\right)\right)
$$

where the conditioned random variable $\mathbf{Y}_{\mathcal{S}_{0: T}}$ is omitted for notational simplicity. Consequently, according to (12), the greedy utility in (15) can be obtained by the precision matrix of the GMRF as

$$
\delta=\frac{1}{2} \log \left(\operatorname{det}\left(\mathbf{Q}_{\overline{\mathcal{S}} \mid \mathcal{S}_{0: T} \cup \mathbf{s}_{n}}\right)\right)-\frac{1}{2} \log \left(\operatorname{det}\left(\mathbf{Q}_{\overline{\mathcal{S}} \mid \mathcal{S}_{0: T}}\right)\right) .
$$

The global optimization is to find the most informative location $\mathbf{s}_{n}^{*}$ from the unsampled sites in $\mathcal{S} \backslash \mathcal{S}_{0: T}$ iteratively. This is achieved by maximizing the utility function $\delta$ over time $T$, under the time constraint $T_{c}$. The experimental design problem is formulated as

$$
\mathbf{s}_{n}^{*}=\underset{\mathbf{s}_{n}}{\operatorname{argmax}} \delta, \text { subject to } \mathbf{s}_{n} \in \mathcal{S} \backslash \mathcal{S}_{0: T}, T \leq T_{c} .
$$

In the proposed framework, the target destinations are generated at the sink by exploiting the GMRF model nonmyopically over the perception field. Concurrently, while heading for these targets, the agents measure the high-information locations myopically along the sampling path via a local greedy search. It is stated that an anytime algorithm can determine increasing better solutions as the runtime increases. The proposed planner is designed as an anytime algorithm, which helps online sampling design and informative path planning in consideration of the computational efficiency and the prediction performance.

The assumptions of the formulated problems are clarified here. First, prior knowledge of an unknown field is learned through an initial exploratory sampling. Second, the field model is time-invariant throughout a sensing cycle, while it may be time-varying in different cycles. Third, the physical quantity at a location does not vary within a sensing cycle, while it may vary over different sensing cycles. Fourth, the measurements and other information can be exchanged between the sink and the agents by medium access control and a multihop routing protocol. In the next section, the proposed planning framework is presented in detail.

\section{INFORMATION-BASED HIERARCHICAL PLANNING}

To properly carry out the online sensing process, an agent should make fast decisions of planning. However, agents in the field generally have limited onboard computational capability, which hinders the implementation of the global MI maximization on it. A sink in an MSN has a superior computational ability compared to the agents in the network. In the proposed hierarchical planner, the target sites are generated over time by the sink and assigned to the mobile sensing agents as their goal destinations.

Information-theoretic global optimization is computationally intensive to find an optimal solution under its NP hardness. As a result, it is practically unable to plan online sampling locations for mobile agents. Myopic greedy search has been proven to be computationally efficient with optimally bounded design [6], which can be developed for online planning of mobile sensing. Therefore, when seeking for upcoming global target sites at the sink, the greedy search can be carried out online at the agents to collect local informative sites. With this idea, a hierarchical planner is developed to simultaneously operate local informative sampling while heading to the global optimal sites that are generated by the global planner.

Specifically, the optimized target sites for an agent $u_{m}$ are generated sequentially over time at the sink, which are pushed in a queue $q_{m}^{(\mathrm{opt})}:=\left(\mathbf{s}_{m, 1}^{\text {(opt) }}, \mathbf{s}_{m, 2}^{\text {(opt) }}, \ldots, \mathbf{s}_{m, j}^{\text {(opt) }}, \ldots\right)$, and then, assigned to the agent. At the same time, the measurement process starts at the agent's initial location $\mathbf{s}_{m, 0}$ at time $t=0$. For the following time steps, the agent heads to the first target 


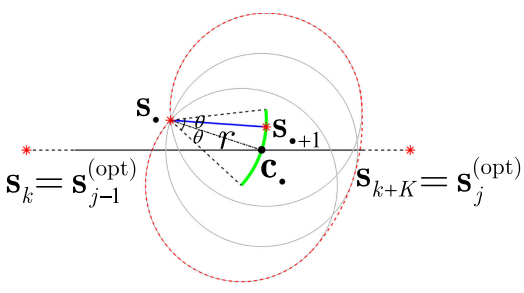

Fig. 2. Search for the next sampling location on the local path.

optimal site, which is designated as $\mathbf{s}_{m, 1}^{(\mathrm{opt})}$. During the travel, the local path is planned on board at the agent by exploiting the local information greedily, which yields a sequence of local sampling locations starting from $\mathbf{s}_{m, 0}$ and ending at $\mathbf{s}_{m, 1}^{\text {(opt) }}$, i.e., $p_{m, 1}=\left(\mathbf{s}_{m, 0}, \mathbf{s}_{m, 1}, \ldots, \mathbf{s}_{m, 1}^{(\mathrm{opt})}\right)$. Subsequently, it heads to the next target site $\mathbf{s}_{m, 2}^{\text {(opt) }}$ from $\mathbf{s}_{m, 1}^{\text {(opt) }}$ with its corresponding sequence of local sampling locations $p_{m, 2}$. Continuing in this manner, the information-driven sampling path can be expressed as $p_{m}=\left(p_{m, 1}, p_{m, 2}, \ldots, p_{m, j}, \ldots\right)$, where $p_{m, j}=\left(\mathbf{s}_{m, k}=\mathbf{s}_{m, j-1}^{\text {(opt) }}, \mathbf{s}_{m, k+1}, \ldots, \mathbf{s}_{m, k+K}=\mathbf{s}_{m, j}^{\text {(opt) }}\right), k \in \mathbb{Z}$, $K \in \mathbb{Z}, j \in \mathbb{Z}^{+}, \mathbf{s}_{m, 0}^{(\text {opt })}=\mathbf{s}_{m, 0}$. For the notational simplicity, the subscript $m$ is omitted in the remaining sections.

\section{A. Local Greedy Search}

The local planner that generates the sampling plan from $\mathbf{s}_{j-1}^{(\mathrm{opt})}$ to $\mathbf{s}_{j}^{\text {(opt) }}$ is introduced in this section. The global planner that obtains the target $\mathbf{s}_{j}^{\text {(opt) }}$ is presented in Section V-B.

Given the starting site $\mathbf{s}_{j-1}^{(\text {opt })}$ and the destination $\mathbf{s}_{j}^{\text {(opt) }}$, a local sampling path is planned on board at the agent. The path is defined by a sequence as $p_{j}=\left(\mathbf{s}_{k}, \mathbf{s}_{k+1}, \ldots, \mathbf{s}_{k+K}\right)$, where $\mathbf{s}_{k}=\mathbf{s}_{j-1}^{\text {(opt) }}, \mathbf{s}_{k+K}=\mathbf{s}_{j}^{\text {(opt) }}$. For any current location $\mathbf{s}_{\bullet}$, the generation of the next sampling location $\mathbf{s}_{\bullet+1}$ on the local sampling path is illustrated in Fig. 2 (the dot in the subscript denotes any location index in the path sequence).

As shown in the figure, the sampling locations are denoted by red stars. To determine the location of $\mathbf{s}_{\bullet+1}$ given $\mathbf{s}_{\bullet}$, a circle is first constructed with the point s. as the circle's center and a radius of $r$. There are two intersections between the circle and the line segment $\mathbf{s}_{j-1}^{\text {(opt) }} \mathbf{s}_{j}^{\text {(opt) }}$, which are denoted as $\mathbf{c}_{1}$ and $\mathbf{c}_{2}$, respectively. Their associate distances to the target site $\mathbf{s}_{j}^{(\mathrm{opt})}$ are obtained as $d_{\mathbf{c} 1}=\left\|\mathbf{s}_{j}^{(\text {opt })}-\mathbf{c}_{1}\right\|$ and $d_{\mathbf{c} 2}=\left\|\mathbf{s}_{j}^{(\text {opt })}-\mathbf{c}_{2}\right\|$, respectively. Among these two intersections, the point that has less distance to $\mathbf{s}_{j}^{(\mathrm{opt})}$ is determined as the target intersection $\mathbf{c}_{\bullet}$, i.e., $\mathbf{c}_{\bullet}=\operatorname{argmin}_{\mathbf{c} \in\left\{\mathbf{c}_{1}, \mathbf{c}_{2}\right\}}\left(\mathrm{d}_{\mathbf{c} 1}, \mathrm{~d}_{\mathbf{c} 2}\right)$. Afterward, an $\operatorname{arc} \mathcal{R}$ is constructed symmetrically with respect to the line segment $\mathbf{s}_{\bullet} \mathbf{c}_{\bullet}$. with a radius of $r$ and an arc angle of $2 \theta$ (the green arc in the figure). The arc is then discretized to a set of points $\widehat{\mathcal{S}}$. Then, the next sampling location $\mathbf{s}_{\bullet+1}$ is generated by greedily selecting the point with the maximum MI among the discretized points on the arc, which can be expressed as

$$
\mathbf{s}_{\bullet}+1=\underset{\mathbf{s}_{n} \in \mathcal{\mathcal { S }}}{\operatorname{argmax}} \operatorname{MI}\left(Y\left(\mathbf{s}_{n}\right) ; \mathbf{Y}_{\left.\mathcal{S}_{\mathcal{A}} \backslash \mathbf{s}_{n}\right)}\right.
$$

where $\widehat{\mathcal{A}}$ defines the surrounding space that is involved by making this observation; $\mathcal{S}_{\widehat{A}}$ denotes the possible sampling locations (mesh vertices) within the area $\widehat{\mathcal{A}}$. The surrounding space $\widehat{\mathcal{A}}$ for $\mathrm{s}_{\bullet+1}$ is indicated in Fig. 2 by the red dash line.
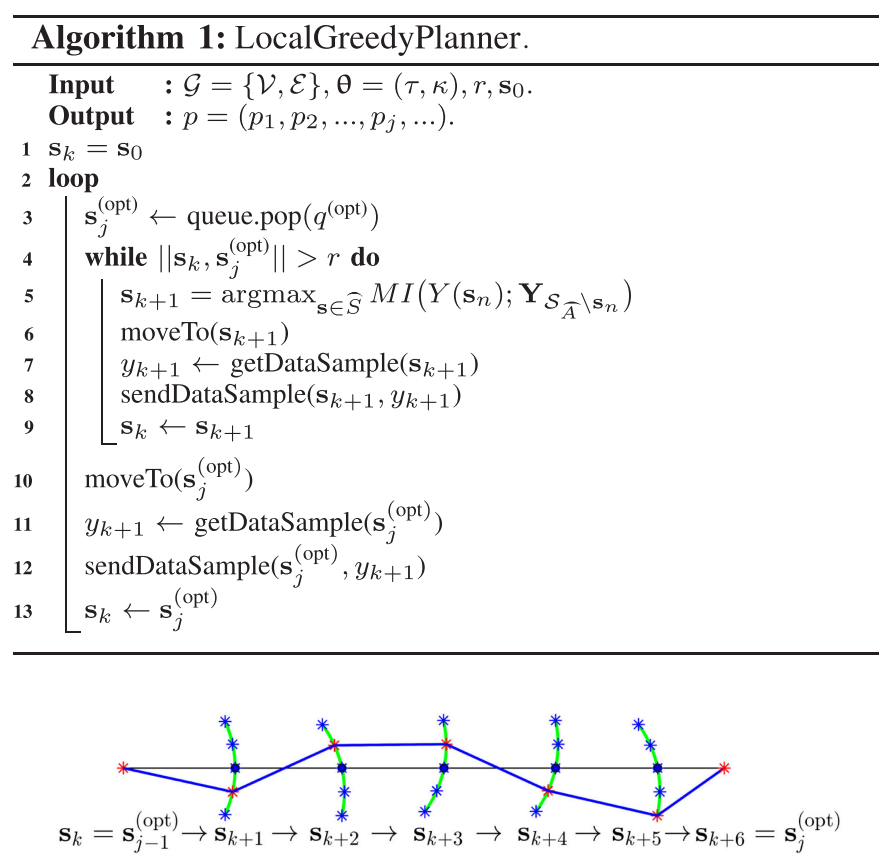

Fig. 3. Execution example of local greedy planning.

The radius $r$ designates the path length between any two consecutive sampling sites, which also indicates the sampling resolution in environmental mapping. The mesh size corresponds to the radius $r$ and remains the same in the local search and the global design. Their relationship is addressed in the global design in Section V-B. An execution example of the local planner is shown in Fig. 3. In the figure, the blue and red stars indicate the discretized potential sampling locations and the selected locations, respectively. The blue line shows the planned local sampling path.

Starting at $\mathbf{s}_{j-1}^{\text {(opt) }}$ and heading to the target $\mathbf{s}_{j}^{(\text {opt })}$, the actions of a mobile sensing agent are listed as follows.

1) Step 1: Start at the current location $\mathbf{s}_{\bullet}$, take a measurement, and exchange information with the sink.

2) Step 2: Check the next target $\mathbf{s}_{j}^{\text {(opt) }}$; if it is located within the radius $r$, i.e., $\left\|\mathbf{s}_{\bullet}, \mathbf{s}_{j}^{(\text {opt })}\right\| \leq r$, set the site $\mathbf{s}_{j}^{(\text {opt })}$ as the next sampling location $\mathrm{s}_{\bullet+1}$; otherwise, go to Step 3 .

3) Step 3: Construct the arc $\mathcal{R}$, discretize the arc to possible sampling points, and then, calculate and set the point corresponding to the maximum $\mathrm{MI}$ as the next sampling location $\mathbf{s}_{\bullet}+1$.

4) Step 4: Move to the next location $\mathbf{s}_{\bullet+1}$; repeat Step 1.

The local planner pseudocode is summarized in Algorithm 1.

\section{B. Global Information-Based Design}

As introduced in Section V-A, an agent operates the sampling mission by visiting the target sites in the order of $q^{(\mathrm{opt})}=$ $\left(\mathbf{s}_{1}^{\text {(opt) }}, \mathbf{s}_{2}^{(\mathrm{opt})}, \ldots, \mathbf{s}_{j}^{\text {(opt) }}, \ldots\right)$. For the optimal design of the target sites that are most informative across the entire field, a global planner is proposed by integrating the overall information at the sink. These target sites are added to the sampling missions of the agents in the field.

In the GMRF model, the optimal design can be derived by finding the locations that have the maximum MI values. A greedy algorithm is implemented to find the optimal site among the potential locations conditioned on the historical sampled 


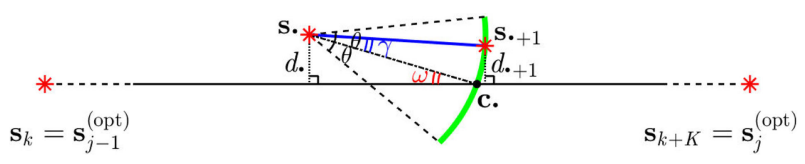

Fig. 4. Next sampling location scheduled by the local planner.

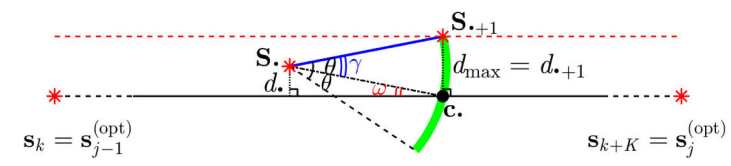

Fig. 5. Bounded area of possible sampling locations.

locations as well as the designed target sites. The generation of the next target site $\mathbf{s}_{j}^{\text {(opt) }}$, starting at time $t=T$, can be expressed as

$$
\mathbf{s}_{j}^{\text {(opt) }}=\underset{\mathbf{s}_{n} \in \mathcal{S} \backslash\left(\mathcal{S}_{0: T} \cup \mathcal{S}_{1: j-1}^{\text {(opt) }}\right)}{\operatorname{argmax}} \operatorname{MI}\left(Y\left(\mathbf{s}_{n}\right) ; \mathbf{Y}_{\mathcal{S} \backslash\left\{\mathcal{S}_{0: T} \cup \mathcal{S}_{1: j-1}^{\text {(opt) }} \cup \mathbf{s}_{n}\right\}}\right)
$$

where $\mathcal{S}_{1: j-1}^{\text {(opt) }}$ denotes the set of previous target sites, with $\mathcal{S}_{1: j-1}^{\text {(opt) }}:=\left\{\mathbf{s}_{1}^{\text {(opt) }}, \mathbf{s}_{2}^{(\mathrm{opt})}, \ldots, \mathbf{s}_{j-1}^{\text {(opt) }}\right\}$. It is noted that when executing to find the next target site $\mathbf{s}_{j}^{(\mathrm{opt})}$ at the sink, the agents take more data samples concurrently. For example, while the sink starts to calculate the next target site $\mathbf{s}_{j}^{\text {(opt) }}$ at time $t=T$, the agents continuously make measurements at time steps $t=T+1, T+2, \ldots$. Consequently, the outcome of $\mathbf{s}_{j}^{(\mathrm{opt})}$ that is derived at time $t=T+K>T$ by (19) does not incorporate the sequential sampling up to the current time, i.e., $S_{1: T+K}$. To solve this problem, a subarea removal strategy is proposed to consider the on-going measuring process when the sink is determining the next target site $\mathbf{s}_{j}^{\text {(opt) }}$.

According to the local planner in Section V-A, the sampling locations are located around the line segment $\mathbf{s}_{j-1}^{\text {(opt) }} \mathbf{s}_{j}^{\text {(opt) }}$. The following proposition can be established.

Proposition 1: The maximum distance from a sampling location s. to the line segment $\mathbf{s}_{j-1}^{\text {(opt) }} \mathbf{s}_{j}^{\text {(opt) }}$ is $d_{\max }=2 r \cdot \sin \frac{\theta}{2}$.

Proof: For a local path $p_{j}$ that is planned in Section V-A, it starts at the target site $\mathbf{s}_{k}=\mathbf{s}_{j-1}^{\text {(opt) }}$ and ends at $\mathbf{s}_{k+K}=\mathbf{s}_{j}^{\text {(opt) }}$. The sequential sampling locations can be derived iteratively, given the current location $s_{\text {. }}$. The auxiliary notations are shown in Fig. 4, where $\omega$ is the angle between $\mathbf{s}_{j-1}^{\text {(opt) }} \mathbf{s}_{j}^{\text {(opt) }}$ and $\mathbf{s}_{\bullet} \mathbf{c}_{\bullet} ; d_{\bullet}$ and $d_{\bullet+1}$ denote the distance from $\mathbf{s}_{\bullet}$ and $\mathbf{s}_{\bullet+1}$ to the line segment $\mathbf{s}_{j-1}^{\text {(opt) }} \mathbf{s}_{j}^{\text {(opt) }}$, respectively. For $\omega \geq 0$, the next local sampling location is expressed as

$$
d_{\bullet+1}=\left\{\begin{array}{l}
r \cdot \sin (\gamma-\omega)+d_{\bullet}, \gamma \in[0, \theta] \\
r \cdot \sin (\omega-\gamma)-d_{\bullet}, \gamma \in[-\theta, 0] .
\end{array} \quad d_{\bullet}=r \cdot \sin \omega\right.
$$

For $\gamma \in[0, \theta], d_{\bullet+1}=r \cdot[\sin (\gamma-\omega)+\sin \omega]$. It is observed that when $\gamma=\theta, \omega=\frac{\theta}{2}$, the maximum distance is $d_{\max } \mid \gamma \in$ $[0, \theta]=2 r \cdot \sin \frac{\theta}{2}$. For $\gamma \in[-\theta, 0], d_{\bullet+1}=r \cdot[\sin (\omega-\gamma)-$ $\sin \omega]$. Also, when $\gamma=-\theta, \omega=0$, the maximum distance is $d_{\max } \mid \gamma \in[-\theta, 0]=r \cdot \sin \theta$. Since $d_{\max } \mid \gamma \in[-\theta, 0]=r$. $\sin \theta=2 r \cdot \sin \frac{\theta}{2} \cdot \cos \frac{\theta}{2} \leq d_{\max } \mid \gamma \in[0, \theta]$, the maximum distance is obtained by $d_{\max }=2 r \cdot \sin \frac{\theta}{2}$. Fig. 5 shows the scenario that $d_{\bullet+1}=d_{\max }$, where the red dash line in the figure indicates

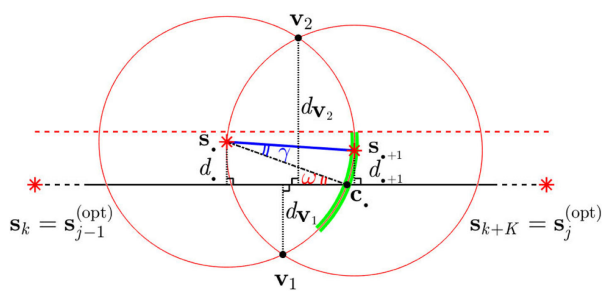

Fig. 6. Two consecutive sampling locations.

the farthest distance to the central line that a local sampling location can achieve.

For $\omega \leq 0$, the sampling location $\mathrm{s}_{\bullet}$ locates beneath the line segment $\mathbf{s}_{j-1}^{\text {(opt) }} \mathbf{s}_{j}^{\text {(opt) }}$. In this case, the results are symmetric to the previous derivations with respect to the line segment. The maximum distance is the same, given by $d_{\max }=2 r \cdot \sin \frac{\theta}{2}$.

Proposition 1 also presents that $\omega_{\max }=\max \omega=$ $\arcsin \frac{d_{\max }}{r}=\arcsin \left(2 \sin \frac{\theta}{2}\right)$. Now, the following lemma can be stated.

Lemma 2: For a local sampling path from $\mathbf{s}_{j-1}^{(\mathrm{opt})}$ to $\mathbf{s}_{j}^{(\mathrm{opt})}$, the sampling locations that are scheduled by the local planner are located within a bounded subarea.

Proof: The result comes directly from Proposition 1. Any sampling location $\mathrm{s}_{\boldsymbol{e}}$ is located at one of the two sides of the line segment $\mathbf{s}_{j-1}^{\text {(opt) }} \mathbf{s}_{j}^{\text {(opt) }}$ within the area, where its maximum distance to the line segment is $d_{\max }=2 r \cdot \sin \frac{\theta}{2}$.

Due to the submodularity property of the sampling process [6], the information in the surrounding area of a sample location is reduced. Since the measuring process is operated along the sampling path, the possible information gain in the area near the local sampling path is reduced. As a result, there is no need to resample this area. In the global planner, the subarea near the local sampling path is excluded when selecting the next target site. The radius $r$ indicates the range of the reduced informative region if the circle center is sensed. It is defined as $r:=\max (\|\mathbf{e}\|), \mathbf{e} \in \mathcal{E}$, since the edges of the triangular mesh specify the neighboring spatial correlations. The following proposition can be stated.

Proposition 3: The informative area that is affected by any two consecutive sampling locations is bounded, with a minimum distance $d_{\min }=2 r \cdot \cos \left(\frac{\pi}{6}-\frac{\theta}{2}+\omega_{\max }\right) \cdot \sin \left(\frac{\pi}{6}-\frac{\theta}{2}\right)$, from the area boundary to the line segment $\mathbf{s}_{j-1}^{(\mathrm{opt})} \mathbf{s}_{j}^{\text {(opt) }}$.

Proof: Fig. 6 shows the scenario of any two consecutive sampling locations, i.e., $\mathbf{s}_{\bullet}$ and $\mathbf{s}_{\bullet+1}$. In the figure, $\gamma$ denotes the angle between the line segments $\mathbf{s}_{\bullet} \mathbf{c}_{\bullet}$ and $\mathbf{s}_{\bullet} \mathbf{s}_{\bullet+1}$. The red circles represent the area with reduced information if the two locations are sampled. $\mathbf{v}_{1}$ and $\mathbf{v}_{2}$ denote the two transect points of the two circles.

Then, the minimal distances $d_{\mathbf{v}_{1}}^{*}=\min d_{\mathbf{v}_{1}}$ and $d_{\mathbf{v}_{2}}^{*}=$ min $d_{\mathbf{v}_{2}}$ can be obtained by simple calculations as follows (the derivation is given in the Appendix):

$$
\begin{aligned}
& d_{\mathbf{v}_{1}}^{*}=2 r \cdot \cos \left(\frac{\pi}{6}-\frac{\theta}{2}+\omega_{\max }\right) \cdot \sin \left(\frac{\pi}{6}-\frac{\theta}{2}\right) \\
& d_{\mathbf{v}_{2}}^{*}=\left\{\begin{array}{l}
d_{\mathbf{v}_{2}}^{(1)}=2 r \cdot \cos \left(\frac{\pi}{6}-\frac{\theta}{2}\right) \cdot \sin \left(\frac{\pi}{6}-\frac{\theta}{2}\right) \text { or } \\
d_{\mathbf{v}_{2}}^{(2)}=2 r \cdot \cos \left(\frac{\pi}{6}-\frac{\theta}{2}-\omega_{\max }\right) \cdot \sin \left(\frac{\pi}{6}-\frac{\theta}{2}\right) .
\end{array}\right.
\end{aligned}
$$

Since $\omega_{\max }=\arcsin \left(2 \sin \frac{\theta}{2}\right)$, it can be determined that $d_{\mathbf{v}_{1}}^{*} \leq d_{\mathbf{v}_{2}}^{*}$ as well as $d_{\mathbf{v}_{1}}^{*} \leq d_{\mathbf{v}_{2}}^{(2)}$. Therefore, by sampling at any two consecutive locations, two circles indicate the reduced 


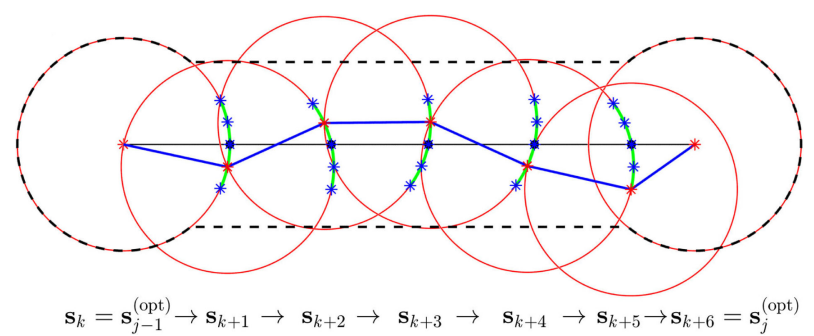

Fig. 7. Dumbbell-shaped area.

information area that they covered, which has a minimum occupied area. The minimum distance from the area boundary to the line segment $\mathbf{s}_{j-1}^{(\mathrm{opt})} \mathbf{s}_{j}^{(\mathrm{opt})}$ is $d_{\min }=d_{\mathbf{v}_{1}}^{*}=2 r \cdot \cos \left(\frac{\pi}{6}-\frac{\theta}{2}+\right.$ $\left.\omega_{\max }\right) \cdot \sin \left(\frac{\pi}{6}-\frac{\theta}{2}\right)$.

According to Proposition 3, there is a region between any two consecutive sampling locations, which becomes less informative after these two positions are observed, no matter where they are located away from the line segment $\mathbf{s}_{j-1}^{\text {(opt) }} \mathbf{s}_{j}^{(\mathrm{opt})}$. Therefore, the following theorem can be stated.

Theorem 4: Any local sampling path from $\mathbf{s}_{j-1}^{(\mathrm{opt})}$ to $\mathbf{s}_{j}^{\text {(opt) }}$ covers a dumbbell-shaped area $\mathcal{A}_{j-1, j}^{\text {(opt) }}$ where the corresponding region has less information if the sampling mission along the local path is completed.

Proof: The result comes directly from Proposition 3. The minimum reduced information area that is covered by any local sampling path is bounded in a dumbbell shape with three main regions, as the dashed line shown in Fig. 7. The two circles at the two ends respond to the sampled locations $\mathbf{s}_{j-1}^{\text {(opt) }}$ and $\mathbf{s}_{j}^{\text {(opt) }}$. The middle rectangular region responds to the sampled locations along the path, which has the minimum distance $d_{\text {min }}$ from the boundary to the line segment $\mathbf{s}_{j-1}^{\text {(opt) }} \mathbf{s}_{j}^{(\mathrm{opt})}$.

When generating the next target site $\mathbf{s}_{j}^{\text {(opt) }}$, the reduced information region defined by the previous sampling paths $\mathcal{A}_{0: j-1}^{\text {(opt) }}:=$ $\mathcal{A}_{0,1}^{\text {(opt) }} \cup \cdots \cup \mathcal{A}_{j-2, j-1}^{\text {(opt) }}$ should be removed from the entire space of interest. This process is defined as the subarea removal (SAR) strategy. By implementing SAR, the generation of the next target site $\mathbf{s}_{j}^{\mathrm{opt}}$, starting at time $t=T$, can be expressed as

$$
\mathbf{s}_{j}^{\text {(opt) }}=\underset{\mathbf{s}_{n} \in \mathcal{S} \backslash \mathcal{S}}{\operatorname{argmax}} \operatorname{MI}\left(Y\left(\mathbf{s}_{n}\right) ; \mathbf{Y}_{\mathcal{S} \backslash\left\{\mathcal{S} \cup \mathbf{s}_{n}\right\}}\right)
$$

where $\mathcal{S} \subseteq \mathcal{A}_{0: j-1}^{\text {(opt) }}$ denotes the potential sampling locations within the removal area $\mathcal{A}_{0: j-1}^{\text {(opt) }}$. After obtaining the target site $\mathbf{s}_{j}^{\text {(opt) }}$, it is assigned to the agent $u^{*}$ for which the last target site of its sampling mission has the shortest distance to the generated site $\mathbf{s}_{j}^{\text {(opt) }}$. By following the proposed framework, the high-level planner at the sink obtains globally informative targets while the agents in the field carry out local search simultaneously. With the continuous running of global planning, the perception area of interest that is left to determine the target sites becomes smaller, which leads to faster execution of the optimal design over time. The pseudocode of the proposed nonmyopic planner is given in Algorithm 2.

\section{NUMERICAL EXPERIMENTS AND DISCUSSION}

In this section, the proposed hierarchical planner is studied through numerical experiments using both synthetic and realworld datasets to evaluate its algorithm performance.
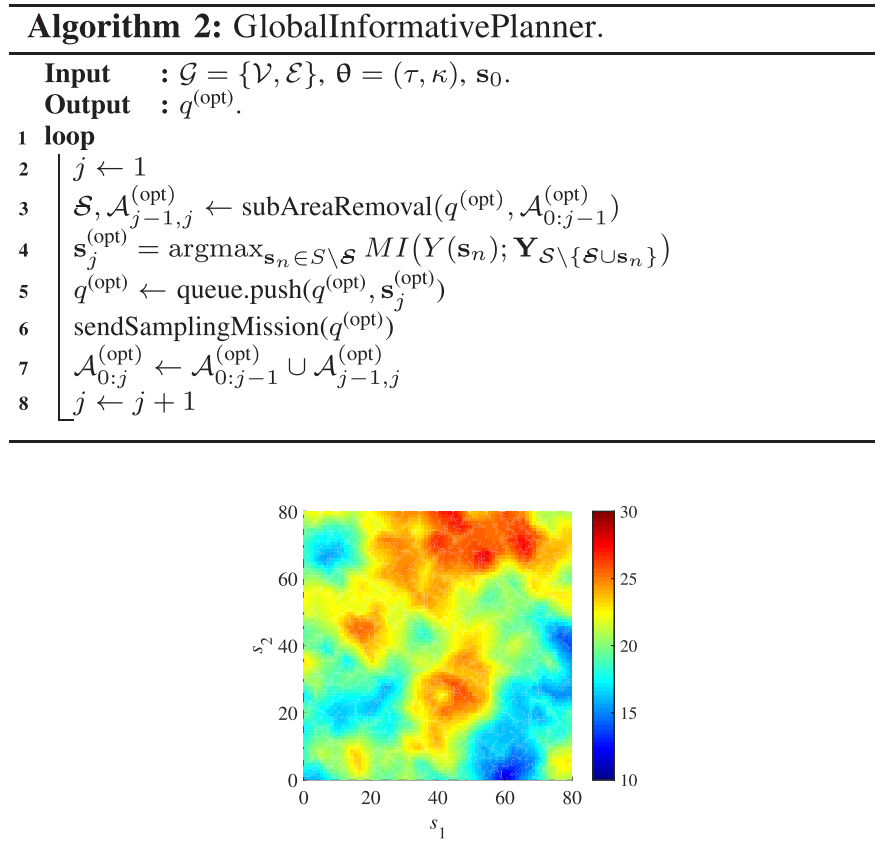

Fig. 8. Numerically generated spatial field.

\section{A. Synthetic Dataset}

The numerically generated spatial field is used in this simulation. The physical quantity of the spatial field is designated on a grid of size $80 \times 80$. The mean function is set at $\boldsymbol{\mu}=20$. The hyperparameters of the precision matrix are chosen as $\boldsymbol{\theta}=(\tau, \kappa)=(1,0.1)$ and the noise level is set at $\sigma_{\epsilon}^{2}=0.2$. With the present configuration, the marginal standard deviation and the range of the Matern covariance function can be obtained as $\sigma=3$ and $\rho=30$, respectively. The generated spatial field is shown in Fig. 8, which is used as the ground truth.

The spatial field is discretized by a triangle mesh using the INLA package in $\mathrm{R}$ [29]. To design the most informative sites in the global planner, the spatial field is discretized on the triangular mesh by approximately 350 vertices of triangles. The sampling location is selected in each local planning stage out of ten discretized locations on the arc. Three mobile sensor agents $(M=3)$ are assigned to make observations, starting from the initial locations, which are randomly selected as $\mathbf{s}_{1,0}=(18,27)$, $\mathbf{s}_{2,0}=(57,61), \mathbf{s}_{3,0}=(71,15)$. The travel distance of the agent between two consecutive samples is set at $r=3$, the agent speed is set at $v=0.4 \mathrm{~m} / \mathrm{s}$, and the tractive effort is set at $F=100 \mathrm{~N}$. The measurement time at a sampling location is set at $t_{M}=10 \mathrm{~s}$. The simulation is executed in $\mathrm{R}$ on a desktop computer with an Intel Core i7-6700K $4 \mathrm{GHz}$ processor and $32 \mathrm{~GB}$ of RAM.

The proposed information-based hierarchical planner that incorporates the subarea removal strategy (IHP-SAR) is evaluated using the numerically generated scalar field. In addition, a distributed planner via local greedy searching (LGS) and an information-based global planner (IGP) for MI optimization, and a Monte Carlo sampling planner are compared in regard to algorithm performance of field mapping. The LGS method operates distributed mobile sensing by exchanging information among agents in the field without a centralized sink. At each agent, the received information from other agents is utilized to update the posterior spatial field model. Afterward, the next sampling site is generated by finding the sampling location that 


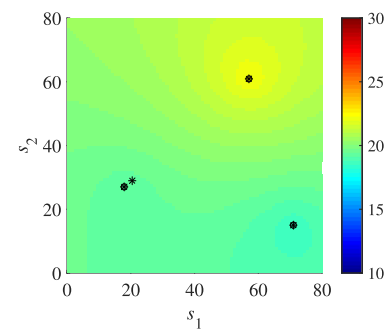

(a)

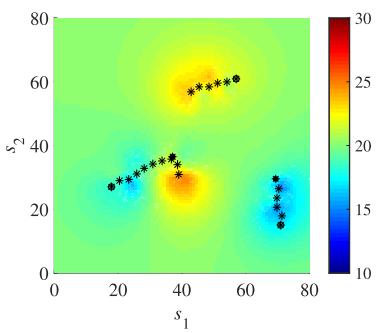

(b)

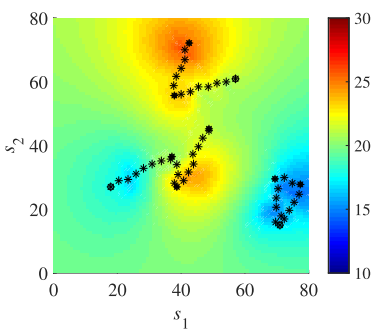

(c)

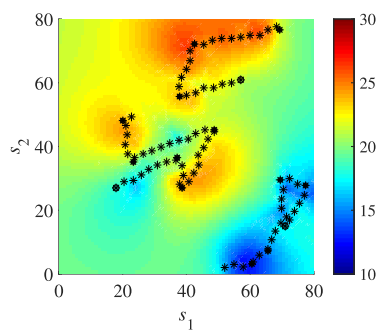

(d)

Fig. 9. Mapping results of the proposed IHP-SAR planner at different times (in seconds). (a) $100 \mathrm{~s}$. (b) $300 \mathrm{~s}$. (c) $600 \mathrm{~s}$. (d) $900 \mathrm{~s}$.

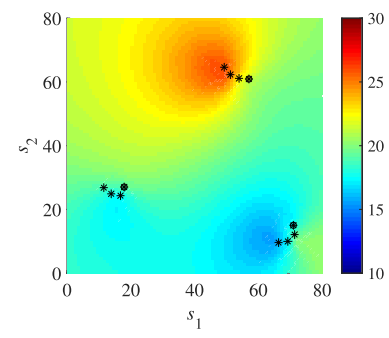

(a)

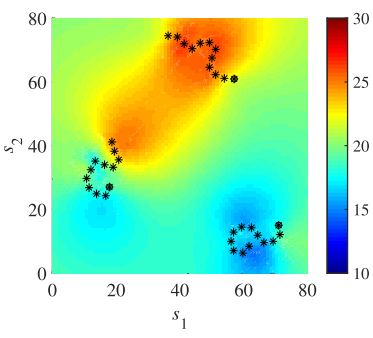

(b)

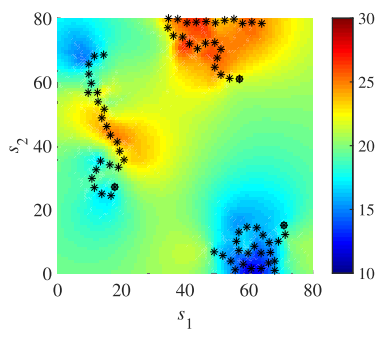

(c)

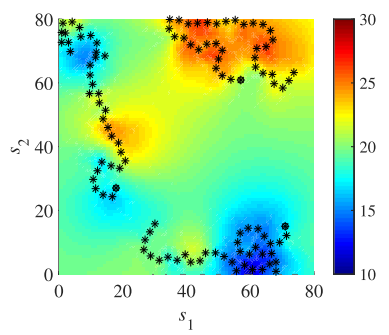

(d)

Fig. 10. Mapping results of the LGS planner at different times (in seconds). (a) 100 s. (b) 300 s. (c) 600 s. (d) $900 \mathrm{~s}$.

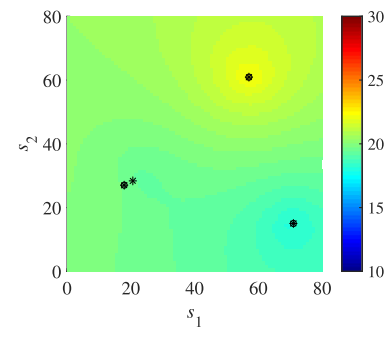

(a)

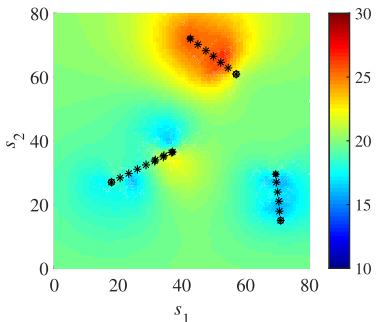

(b)

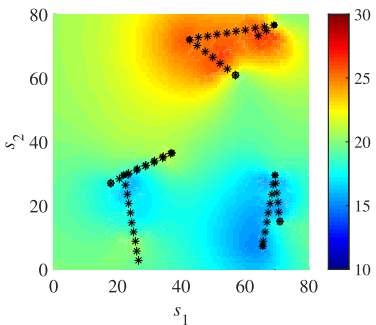

(c)

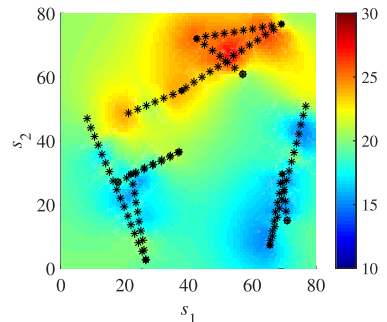

(d)

Fig. 11. Mapping results of the IGP planner at different times (in seconds). (a) $100 \mathrm{~s}$. (b) $300 \mathrm{~s}$. (c) $600 \mathrm{~s}$. (d) $900 \mathrm{~s}$.

is the most informative locally. This strategy has been studied in past works (e.g., [15], [22], [16]) to achieve online planning for adaptive sampling and field mapping. Specifically, a circular arc (with the current location as the circle center) is discretized to multiple points, among which, the most informative site is selected as the next sampling location. The Monte Carlo sampling approach directly selects a point randomly on the arc. The IGP method optimizes the design of the target sites globally using the GMRF scheme, which ranks all possible sites based on their MI quantities, and then, selects the best ones as the sites to be visited. The agents take samples while moving toward the targets.

Prediction outcomes are updated with time. The resulting predictions of the spatial field, by implementing the proposed and the compared algorithms, are shown in Figs. 9-12 (at times $t=100,300,600$, and $900 \mathrm{~s}$, respectively). In the figures, the sampled locations are shown using star markers. The colors illustrate the physical quantities over the scalar field. As shown, the resulting predictions become closer to the ground truth as the time increases.
RMSE is implemented as the measure to evaluate the prediction results, which is defined as

$$
\operatorname{RMSE}(t)=\sqrt{\frac{1}{|I|} \Sigma_{\mathbf{i} \in I}\left[\hat{Y}(\mathbf{i}) \mid \mathbf{y}_{1: t}-y(\mathbf{i})\right]^{2}}
$$

where $I$ represents the set of the grid vertices for spatial interpolation, and $y(\mathbf{i})$ captures the ground truth value at a location $\mathbf{i} \in I$. Fig. 13 shows the prediction performance of the RMSE variation over time and the energy cost. As can be seen, the trend of the RMSE results reduces gradually with increased observations over time and energy consumption.

Fig. 13(a) shows that the LGS and the random sampling can provide better performance in the initial stage of the sampling process. This is because these approaches are able to rapidly issue the sampling sites and start to guide the agents for data collection. In comparison, the IGP and the proposed IHP-SAR approaches have to calculate the first target site before the agents can start to take data samples. As time progresses, the LGS or random sampling may remain trapped in a small region for some period of the sampling mission [see Figs. 10(d) and 12(d)]. Without global planning, the LGS method requires more 


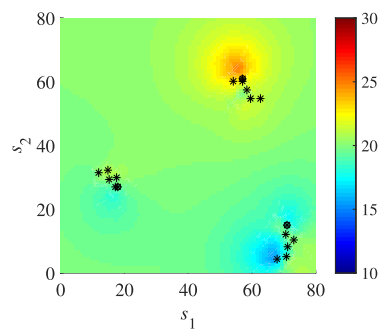

(a)

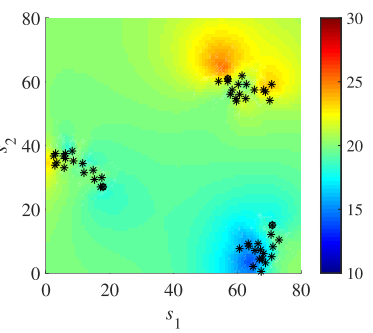

(b)

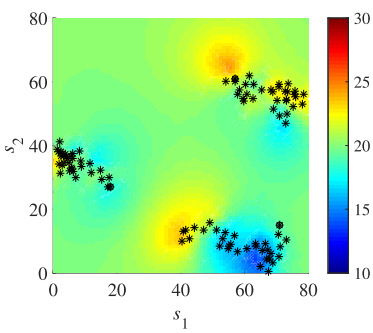

(c)

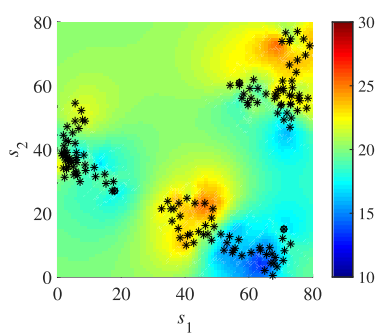

(d)

Fig. 12. Mapping results of the random sampling at different times (in seconds). (a) 100 s. (b) 300 s. (c) 600 s. (d) 900 s.

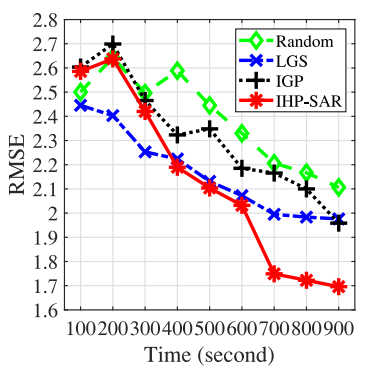

(a)

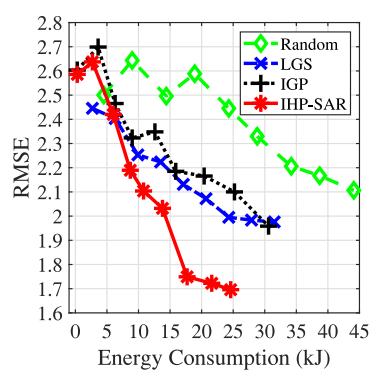

(b)
Fig. 13. Algorithm performance of the RMSE results.

information exchange among agents to establish the field model on board each agent. In addition, it operates local greedy search to ensure online planning for mobile sensing. Thus, this distributed planner leads to myopic exploration with its degraded mapping results over time. In comparison, the proposed IHPSAR method effectively navigates the mobile agents to globally informative targets in a nonmyopic manner. The IGP method covers the targets directly but without searching for the local high-information locations. In comparison, IHP-SAR achieves superior prediction results since it provides an online solution by effectively integrating myopic and nonmyopic informationbased planning.

Energy is consumed over time in the planned sampling mission. To evaluate the planning performance on resulting energy consumption, the cost is simulated as $W(t)=F \cdot d=F \cdot v \cdot t$, where $F$ denotes the tractive effort of the agent, $d$ denotes the travel distance, $v$ denotes the agent speed, and $t$ denotes the travel time duration. In the experiments, at the speed of $0.4 \mathrm{~m} / \mathrm{s}$, the agents carried out the planned mission progressively within the sensing cycle $T_{c}=900 \mathrm{~s}$. During the mission operation, the agents visited more planned sites and traveled longer distance, which leads to more energy consumption. Fig. 13(b) demonstrates that the proposed IHP-SAR provides superior prediction but at a lower energy cost when compared with the other approaches.

Global design provides nonmyopically designed targets but intensively requires computational resources. Local search drastically facilitates computational efficiency, yet it leads to myopic local optimal solution. Accordingly, there is a natural conflict between computational efficiency and mapping accuracy for online planning of mobile sensing. To further discuss the experiments, several metrics are considered, including:

1) $\bar{\delta}$ : average utility gain per designed sampling site;

2) $\bar{N}$ : average number of designed sampling site per $100 \mathrm{~s}$;

3) $\Delta \mathrm{RMSE}_{t}$ : average RMSE decrease per $100 \mathrm{~s}$;
TABLE I

ALGORITHM PERFORMANCE IN THE EXPERIMENTS

\begin{tabular}{|c||c|c|c|c|}
\hline Metric & Random & LGS & IGP & IHP-SAR \\
\hline \hline $\bar{\delta}$ & N/A & 2.66 & 9.45 & 8.40 \\
\hline $\bar{N}$ & 16.33 & 11.67 & 1.44 & 9.11 \\
\hline$\Delta R M S E_{t}$ & $4.92 \mathrm{E}-2$ & $5.62 \mathrm{E}-2$ & $8.07 \mathrm{E}-2$ & $\mathbf{1 1 . 1 3 E - 2}$ \\
\hline$\Delta R M S E_{e}$ & $8.74 \mathrm{E}-4$ & $13.50 \mathrm{E}-4$ & $24.49 \mathrm{E}-4$ & $\mathbf{3 5 . 3 4 E - 4}$ \\
\hline
\end{tabular}

N/A denotes no informative design in random sampling.

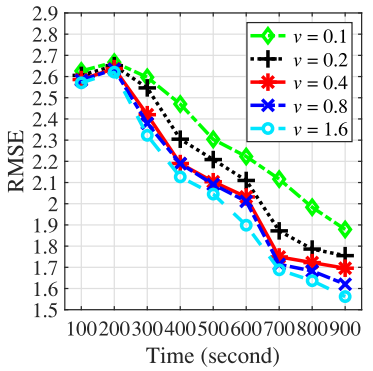

(a)

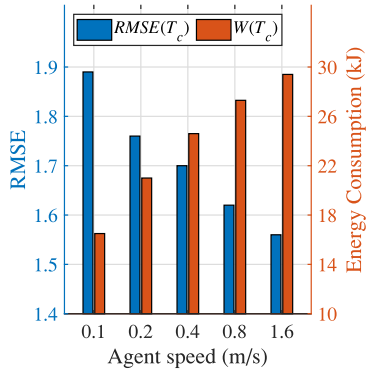

(b)
Fig. 14. IHP-SAR performance on different agent speeds.

4) $\Delta \mathrm{RMSE}_{e}$ : average RMSE decrease per kilojoule.

The experimental results of the compared algorithms are given in Table I. The larger values of the metrics 3 and 4 represent better online mapping results. As verified in the table, IGP provides the best utility gain for each designed sample but obtains the lowest number of target sites. LGS efficiently generates sampling locations for mobile sensing agents. However, the utility gain for each sample is the lowest. In comparison, the proposed IHP-SAR method trades off global planning and local planning through the developed SAR mechanism. The experimental results of $\Delta \mathrm{RMSE}_{t}$ and $\Delta \mathrm{RMSE}_{e}$ demonstrate its best performance on reducing mapping error per time as well as per energy consumption.

To evaluate the algorithm performance corresponding to agent mobility, experiments are conducted with respect to different speeds. Specifically, the agent speed $v$ is varied and set to $\{0.1$, $0.2,0.4,0.8,1.6\}$ in units of meter per second $(\mathrm{m} / \mathrm{s})$. The results of the proposed method are shown in Fig. 14. Fig. 14(a) shows the RMSE results over time under different agent speeds. It implies that a higher moving speed leads to a better exploration result for mapping. This is due to the fact that a faster agent can visit more target sites within a sensing cycle.

In general, more observations at the target sites lead to more accurate mapping results. However, visiting more sites consumes more power, as a result of a longer travel distance in 


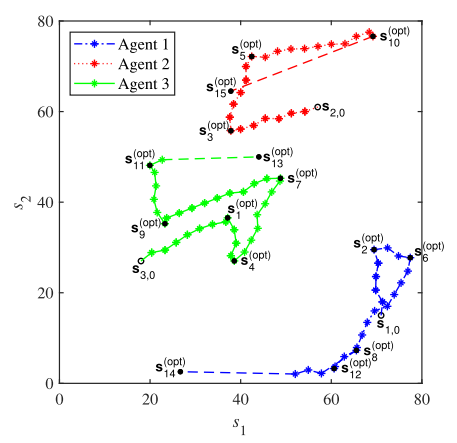

Fig. 15. IHP-SAR sampling mission at $t=900 \mathrm{~s}$.

TABLE II

TARGET SITES AND THEIR CORRESPONDING ASSIGNMENTS

\begin{tabular}{|c|c|c|c|c|c|c|}
\hline \multicolumn{2}{|c|}{ Target Site } & \multicolumn{2}{c|}{ Algorithm Runtime } & \multicolumn{2}{c|}{ Sampling Mission } \\
\hline Site & Coordinates & $\begin{array}{c}\text { Start } \\
\text { (second) }\end{array}$ & $\begin{array}{c}\text { End } \\
\text { (second) }\end{array}$ & $\begin{array}{c}\text { Duration } \\
\text { (second) }\end{array}$ & $\begin{array}{c}\text { Assigned } \\
\text { Agent }\end{array}$ & $\begin{array}{c}\text { Arrival } \\
\text { (second) }\end{array}$ \\
\hline \hline $\mathbf{s}_{1}^{\text {(opt) }}$ & $(37.13,36.54)$ & 0 & 67.62 & 67.62 & $u_{3}$ & 218.82 \\
\hline $\mathbf{s}_{2}^{\text {(opt) }}$ & $(69.44,29.52)$ & 67.62 & 132.06 & 64.44 & $u_{1}$ & 225.96 \\
\hline $\mathbf{s}_{3}^{\text {(opt) }}$ & $(37.84,55.76)$ & 132.06 & 195.20 & 63.14 & $u_{2}$ & 327.30 \\
\hline $\mathbf{s}_{4}^{\text {(opt) }}$ & $(38.59,27.03)$ & 195.20 & 257.54 & 62.34 & $u_{3}$ & 332.34 \\
\hline $\mathbf{s}_{5}^{\text {(opt) }}$ & $(42.44,72.16)$ & 257.54 & 319.82 & 62.28 & $u_{2}$ & 440.30 \\
\hline $\mathbf{s}_{6}^{\text {(oppt) }}$ & $(77.43,27.74)$ & 319.82 & 381.62 & 61.80 & $u_{1}$ & 437.32 \\
\hline $\mathbf{s}_{7}^{\text {(opt) }}$ & $(48.78,45.28)$ & 381.62 & 443.09 & 61.47 & $u_{3}$ & 594.29 \\
\hline $\mathbf{s}_{\mathbf{8}}^{\text {(opt) }}$ & $(65.54,7.28)$ & 443.09 & 504.20 & 61.11 & $u_{1}$ & 674.50 \\
\hline $\mathbf{s}_{9}^{\text {(opt) }}$ & $(23.28,35.25)$ & 504.20 & 563.97 & 59.77 & $u_{3}$ & 783.69 \\
\hline $\mathbf{s}_{10}^{\text {(opp) }}$ & $(69.20,76.61)$ & 563.97 & 621.88 & 57.91 & $u_{2}$ & 811.28 \\
\hline $\mathbf{s}_{11}^{\text {(opt) }}$ & $(19.96,48.13)$ & 621.88 & 678.49 & 56.61 & $u_{3}$ & 877.59 \\
\hline $\mathbf{s}_{12}^{\text {(opt) }}$ & $(60.62,3.27)$ & 678.49 & 734.42 & 55.93 & $u_{1}$ & 790.12 \\
\hline $\mathbf{s}_{13}^{\text {(opt) }}$ & $(44.00,50.00)$ & 734.42 & 789.95 & 55.53 & $u_{3}$ & N/A \\
\hline $\mathbf{s}_{14}^{\text {(oppt) }}$ & $(26.69,2.57)$ & 789.95 & 844.60 & 54.65 & $u_{1}$ & N/A \\
\hline $\mathbf{s}_{15}^{\text {(opt) }}$ & $(37.83,64.51)$ & 844.60 & 897.85 & 53.25 & $u_{2}$ & N/A \\
\hline
\end{tabular}

N/A denotes that the target sites have not been visited within a sensing cycle.

a sensing cycle. To further demonstrate this issue, Fig. 14(b) provides the final mapping accuracy $\operatorname{RMSE}\left(T_{c}\right)$ and energy consumption $W\left(T_{c}\right)$ after finishing the planned mission, with respect to different speeds. The speed settings $v=0.1,0.2$, $0.4,0.8,1.6 \mathrm{~m} / \mathrm{s}$ result in associated final error performance as $\operatorname{RMSE}\left(T_{c}\right)=1.89,1.76,1.70,1.62,1.56$, with the energy consumption as $W\left(T_{c}\right)=16.5,21.0,24.6,27.3,29.4 \mathrm{~kJ}$, respectively. The experimental results verify that a higher speed leads to a lower final RMSE, but costing more energy.

Remark 6: The proposed IHP-SAR algorithm generates the global design in an anytime planning manner. The processing time of finding the next target site decreases as the number of measurements increases.

This remark can be examined through the experimental results. Fig. 15 and Table II illustrate the operation outcomes by implementing the proposed approach at time $t=900 \mathrm{~s}$. The figure shows the order in which the target sites were generated and their assignment conditions for the three agents. The black dots, star markers, and the line segments indicate the generated target sites, sampled locations, and the traveled path, respectively. The target sites are labeled in the order of their generation time. Table II summarizes the processing time for generating the target sites and the planned sampling mission. It is seen that the sites have been obtained persistently, which could also be interrupted at any time, providing the associated solutions up to that time. Also, they were derived increasingly faster, taking $67.62 \mathrm{~s}$ for the first site and reducing to $53.25 \mathrm{~s}$ for the last one, because the size of the potential sites on the mesh decreases over time

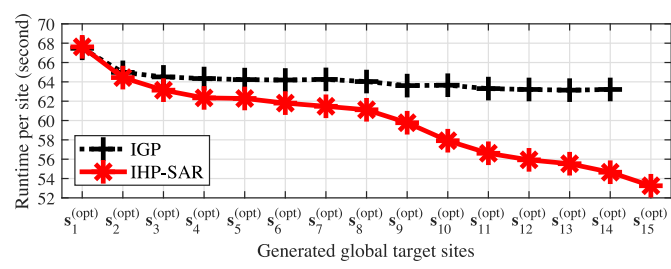

Fig. 16. Algorithm runtime for finding global target sites.

TABLE III

HYPERPARAMETERS OF THE REAL-WORLD DATASET

\begin{tabular}{|c||c|c|c|c||c|c|c|c|}
\hline Time & 3630 & 3660 & 3690 & 3720 & 4260 & 4290 & 4320 & 4350 \\
\hline \hline$\sigma^{2}$ & 1.86 & 1.59 & 1.30 & 1.48 & 0.69 & 0.62 & 0.51 & 0.65 \\
\hline$\rho$ & 39.22 & 36.32 & 32.65 & 35.11 & 19.55 & 17.90 & 17.18 & 19.49 \\
\hline$\tau$ & 4.06 & 4.06 & 4.04 & 4.07 & 3.43 & 3.21 & 3.39 & 3.41 \\
\hline$\kappa$ & 0.07 & 0.08 & 0.09 & 0.08 & 0.14 & 0.16 & 0.16 & 0.15 \\
\hline
\end{tabular}

through the SAR strategy. As expected, the planner determines the most informative site one by one, and the runtime of finding the next target decreases over time with more data samples. The experimental results support the concluding statement in remark 6.

The IGP method and the proposed IHP-SAR method operate global planning at the sink. The associated overhead to obtain the global information mainly comes from computational cost. Their algorithm runtime for finding the global target sites is compared and shown in Fig. 16. In the figure, $x$-axis indicates the global target sites in their generation order; $y$-axis indicates their associated execution time per site. As shown in the figure, the runtime per generated global optimal site of the IGP method remains relatively unchanged. Within the sensing cycle $900 \mathrm{~s}$, it generates 14 global informative sites with the average time cost of $64.15 \mathrm{~s}$. In comparison, the proposed IHP-SAR method generates 15 optimal sites with the average time cost of $59.86 \mathrm{~s}$. The proposed method can effectively and increasingly shorten the execution time for generating global informative sites. At the mobile agents, the average runtime of IHP-SAR for generating a local informative site is $1.52 \mathrm{~s}$. This runtime at mobile agents enables online navigation of mobile sensing.

\section{B. Real-World Dataset}

The proposed planning algorithm is also implemented using a real-world dataset from the Intel Berkeley Research Lab [30] to evaluate its performance. In the experiment, the indoor temperature is selected as the monitored environmental quantity. The GPR results, by making use of the observations at the 54 sensor nodes in the dataset, are used as the ground truth of the perception field. The hyperparameters of the environmental model may remain the same or vary over time. Table III presents the examples of the hyperparameter values at different time stamps, which indicates that the model remains the same in some time periods but may change in some other time stamps (as assumed in Section IV). Fig. 17(a) and (b) shows the ground truth maps of these two models at the time stamp 3630 and 4260, respectively.

In the experiment, at the initial deployment, an exploration process utilizing the information-driven approach in the work of [16] is first implemented to learn the prior knowledge of the study field. After determining the hyperparameters of the environmental model, the proposed planner is used to exploit the 


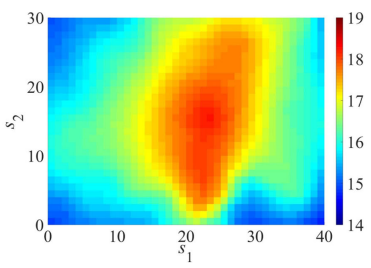

(a)

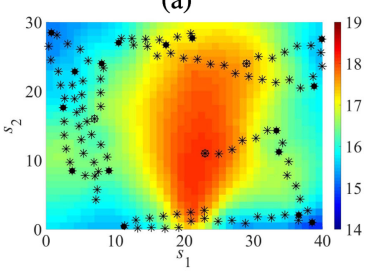

(c)

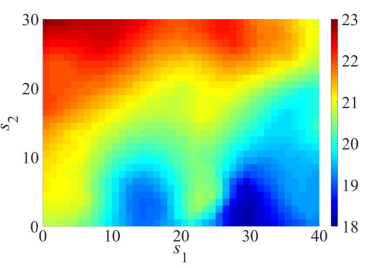

(b)

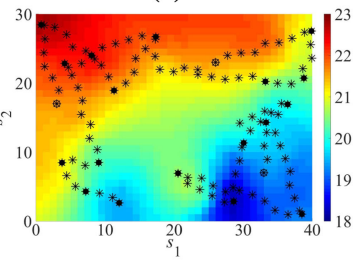

(d)
Fig. 17. Mapping results on the real-world dataset.

environment for sampling design and path planning. Three mobile sensing agents are implemented starting at the randomly predetermined locations $\mathbf{s}_{1,0}=(39,11), \mathbf{s}_{2,0}=(45,75), \mathbf{s}_{3,0}=$ $(6,70)$. Each sensing cycle is specified as $15 \mathrm{~min}$. Fig. 17(c) and (d) shows the predicting maps at the time stamp 3630 and 4260, respectively, by implement the proposed algorithm. RMSE of the time stamps 3630 and 4260 are 0.2282 and 0.2325 , respectively.

In a real-world application, the environmental phenomena may vary in two ways: 1) the physical quantities of the monitored parameters may change over time, but the spatial correlations over the underlying field remain and 2) both physical quantities and spatial correlations may change over time. The former case indicates that the environmental field model is time-invariant, which uses the same optimal sampling design in different sensing cycles. In this case, the agents operate their sampling missions by following the same designed sampling frames over multiple sensing cycles. The latter case indicates that the environmental field model changes and a model updating process is required. With the measurements from the previous sensing cycle, the model hyperparameters can be re-estimated to interpret the variance of the spatial correlation over the random field. The model updating and information-theoretic planning can be operated iteratively to handle a time-varying field model.

It is noted that the developed SAR mechanism does not depend on ongoing local search to operate global design. As a result, the global planning at the sink will not be affected by missing data packets from the agents. However, missing data packets of local collections from the agents may change mapping results. In addition, missing data packets of sampling targets from the sink will cause loss of moving goals for local planning. A check-and-resend mechanism can be implemented to ensure reliable data transmission. For the case that resending fails due to a severely poor network circumstance, the agent will complete the previously assigned mission and stay at the last assigned target to wait for a new target from the sink.

\section{CONCLUSION}

This article proposed a novel hierarchical system framework to plan an information-based sampling mission, which was implemented to carry out adaptive sampling for MSN-based environmental field mapping. The proposed framework provided an effective hierarchical scheme that integrated myopic searching and nonmyopic optimization. The local planner operated onboard planning to plot the high-information locations along the local sampling path. Concurrently, the global planner at the sink, with its beneficial characteristics as an anytime algorithm, determined the subsequent destinations that were the most informative sites over the entire field. A subarea removal strategy was introduced in this article to address the growing set of sequential sampled locations in the field during the global optimization at the sink. The proposed hierarchical framework provides a practical solution for online sampling and field mapping using mobile sensor networks.

\section{APPENDIX}

\section{PROOF OF PROPOSITION 3}

For $\omega \in\left[0, \omega_{\max }=\arcsin \left(2 \sin \frac{\theta}{2}\right)\right], \gamma \in[-\theta, \theta], \theta \in\left[0, \frac{\pi}{4}\right]$, the distance from $\mathbf{v}_{1}$ and $\mathbf{v}_{2}$ to the line segment $\mathbf{s}_{j-1}^{\text {(opt) }} \mathbf{s}_{j}^{\text {(opt) }}$ are expressed, respectively, as

$$
\begin{aligned}
& d_{\mathbf{v}_{1}}=r \cdot \sin \left(\frac{\pi}{3}+\omega-\gamma\right)-r \cdot \sin \omega \\
& d_{\mathbf{v}_{2}}=r \cdot \sin \left(\frac{\pi}{3}-\omega+\gamma\right)+r \cdot \sin \omega .
\end{aligned}
$$

To minimize $d_{\mathbf{v}_{1}}, \gamma^{*}=\operatorname{argmin}_{\gamma} \mathrm{d}_{\mathbf{v}_{1}}=\max \gamma=\theta$. Then, get the derivative $\frac{d}{d \omega} d_{\mathbf{v}_{1}}$ to find $\omega$ such that $d_{\mathbf{v}_{1}}$ is minimized, where $\frac{d}{d \omega} d_{\mathbf{v}_{1}}=r \cdot \cos \left(\frac{\pi}{3}+\omega-\theta\right)-r \cdot \cos \omega$. Since $\theta \leq \frac{\pi}{4}<$ $\frac{\pi}{3}$, it is observed that $\frac{d}{d \omega} d_{\mathbf{v}_{1}}<0$. Thus, $\omega^{*}=\operatorname{argmin}_{\omega} \mathrm{d}_{\mathbf{v}_{1}}=$ $\max \omega=\omega_{\max }$. The minimum $d_{\mathbf{v}_{1}}$ is derived as

$$
\begin{aligned}
\min d_{\mathbf{v}_{1}} & =r \cdot \sin \left(\frac{\pi}{3}+\omega_{\max }-\theta\right)-r \cdot \sin \omega_{\max } \\
& =2 r \cdot \cos \left(\frac{\pi}{6}-\frac{\theta}{2}+\omega_{\max }\right) \cdot \sin \left(\frac{\pi}{6}-\frac{\theta}{2}\right) .
\end{aligned}
$$

Similarly, to minimize $d_{\mathbf{v}_{2}}, \gamma^{*}=\operatorname{argmin}_{\gamma} \mathrm{d}_{\mathbf{v}_{2}}=\min \gamma=$ $-\theta$. Then, get the derivative $\frac{d}{d \omega} d_{\mathbf{v}_{2}}$ to find $\omega$ such that $d_{\mathbf{v}_{2}}$ is minimized, where $\frac{d}{d \omega} d_{\mathbf{v}_{2}}=-r \cdot \cos \left(\frac{\pi}{3}-\theta-\omega\right)+r \cdot \cos \omega$. The minimum $d_{\mathbf{v}_{2}}$ can be obtained when

$$
\omega=\left\{\begin{array}{l}
\omega_{1}=\omega_{\min }=0 \\
\omega_{2}=\omega_{\max }=\arcsin \left(2 \sin \frac{\theta}{2}\right) \\
\omega_{3}=\arg _{\omega}\left(\frac{d}{d \omega} d_{\mathbf{v}_{2}}=0\right)=\frac{\pi}{6}-\frac{\theta}{2} .
\end{array}\right.
$$

Thus, the minimum $d_{\mathbf{v}_{2}}$ can be obtained when

$$
d_{\mathbf{v}_{2}}=\left\{\begin{aligned}
d_{\mathbf{v}_{2}}^{(1)}= & r \cdot \sin \left(\frac{\pi}{3}-\theta\right), & & \omega_{1}=0 \\
d_{\mathbf{v}_{2}}^{(2)}= & r \cdot \sin \left(\frac{\pi}{3}-\theta-\omega_{\max }\right) & & \\
& +r \cdot \sin \omega_{\max }, & & \omega_{2}=\omega_{\max } \\
d_{\mathbf{v}_{2}}^{(3)}= & 2 \cdot r \cdot \sin \left(\frac{\pi}{6}-\frac{\theta}{2}\right), & & \omega_{3}=\frac{\pi}{6}-\frac{\theta}{2} .
\end{aligned}\right.
$$

It is seen that $d_{\mathbf{v}_{2}}^{(1)}=r \cdot \sin \left(\frac{\pi}{3}-\theta\right)=2 r \cdot \sin \left(\frac{\pi}{6}-\frac{\theta}{2}\right)$. $\cos \left(\frac{\pi}{6}-\frac{\theta}{2}\right)<d_{\mathbf{v}_{2}}^{(3)} \quad$ and $\quad d_{\mathbf{v}_{2}}^{(2)}=2 r \cdot \sin \left(\frac{\pi}{6}-\frac{\theta}{2}\right) \cdot \cos \left(\frac{\pi}{6}-\right.$ $\left.\frac{\theta}{2}-\omega_{\max }\right) \leq d_{\mathbf{v}_{2}}^{(3)}$. However, the relationship between $d_{\mathbf{v}_{2}}^{(1)}$ and $d_{\mathbf{v}_{2}}^{(2)}$ is all possible since $\cos \left(\frac{\pi}{6}-\frac{\theta}{2}-\omega_{\max }\right)$ can be bigger than, small than, or equal to $\cos \left(\frac{\pi}{6}-\frac{\theta}{2}\right)$. Thus, $\min d_{\mathbf{v}_{2}}=\min \left\{d_{\mathbf{v}_{2}}^{(1)}, d_{\mathbf{v}_{2}}^{(2)}\right\}$, i.e.,

$$
\min d_{\mathbf{v}_{2}}=\left\{\begin{array}{l}
2 r \cdot \cos \left(\frac{\pi}{6}-\frac{\theta}{2}\right) \cdot \sin \left(\frac{\pi}{6}-\frac{\theta}{2}\right) \text { or } \\
2 r \cdot \cos \left(\frac{\pi}{6}-\frac{\theta}{2}-\omega_{\max }\right) \cdot \sin \left(\frac{\pi}{6}-\frac{\theta}{2}\right) .
\end{array}\right.
$$




\section{REFERENCES}

[1] Y. Wang, R. Tan, G. Xing, X. Tan, J. Wang, and R. Zhou, "Spatiotemporal aquatic field reconstruction using cyber-physical robotic sensor systems," ACM Trans. Sensor Netw., vol. 10, no. 4, 2014, Art. no. 57.

[2] P. Tokekar, J. Vander Hook, D. Mulla, and V. Isler, "Sensor planning for a symbiotic UAV and UGV system for precision agriculture," IEEE Trans. Robot., vol. 32, no. 6, pp. 1498-1511, Dec. 2016.

[3] N.-B. Chang, K. Bai, S. Imen, C.-F. Chen, and W. Gao, "Multisensor satellite image fusion and networking for all-weather environmental monitoring," IEEE Syst. J., vol. 12, no. 2, pp. 1341-1357, Jun. 2018.

[4] K. L.-M. Ang, J. K. P. Seng, and A. M. Zungeru, "Optimizing energy consumption for big data collection in large-scale wireless sensor networks with mobile collectors," IEEE Syst. J., vol. 12, no. 1, pp. 616-626, Mar. 2018.

[5] V. V. Fedorov and P. Hackl, Model-Oriented Design of Experiments (Lecture Notes in Statistics, vol. 125). New York, NY, USA: Springer-Verlag, 2012.

[6] A. Krause, A. Singh, and C. Guestrin, "Near-optimal sensor placements in Gaussian processes: Theory, efficient algorithms, and empirical studies," J. Mach. Learn. Res., vol. 9, pp. 235-284, 2008.

[7] C.-W. Ko, J. Lee, and M. Queyranne, "An exact algorithm for maximum entropy sampling," Oper. Res., vol. 43, no. 4, pp. 684-691, 1995.

[8] L. Csató and M. Opper, "Sparse on-line Gaussian processes," Neural Comput., vol. 14, no. 3, pp. 641-668, 2002.

[9] H. Rue and L. Held, Gaussian Markov Random Fields: Theory and Applications. Boca Raton, FL, USA: CRC Press, 2005.

[10] L. V. Nguyen, S. Kodagoda, R. Ranasinghe, and G. Dissanayake, "Mobile robotic wireless sensor networks for efficient spatial prediction," in Proc. IEEE/RSJ Int. Conf. Intell. Robots Syst., 2014, pp. 1176-1181.

[11] Y. Xu, J. Choi, S. Dass, and T. Maiti, Bayesian Prediction and Adaptive Sampling Algorithms for Mobile Sensor Networks: Online Environmental Field Reconstruction in Space and Time. Berlin, Germany: Springer, 2015.

[12] G. Fernández-Avilés et al., Spatial and Spatio-Temporal Geostatistical Modeling and Kriging. Hoboken, NJ, USA: Wiley, 2015.

[13] F. Lindgren, H. Rue, and J. Lindström, "An explicit link between Gaussian fields and Gaussian Markov random fields: The stochastic partial differential equation approach," J. Roy. Statist. Soc., Ser. B (Statist. Methodology), vol. 73, no. 4, pp. 423-498, 2011.

[14] Y. Xu and J. Choi, "Spatial prediction with mobile sensor networks using Gaussian processes with built-in Gaussian Markov random fields," Automatica, vol. 48, no. 8, pp. 1735-1740, 2012.

[15] Y. Xu, J. Choi, S. Dass, and T. Maiti, "Efficient Bayesian spatial prediction with mobile sensor networks using Gaussian Markov random fields," Automatica, vol. 49, no. 12, pp. 3520-3530, 2013.

[16] L. V. Nguyen, S. Kodagoda, R. Ranasinghe, and G. Dissanayake, "Information-driven adaptive sampling strategy for mobile robotic wireless sensor network," IEEE Trans. Control Syst. Technol., vol. 24, no. 1, pp. 372-379, Jan. 2016

[17] E. Kreuzer and E. Solowjow, "Learning environmental fields with micro underwater vehicles: A path integral-Gaussian Markov random field approach," Auton. Robots, vol. 42, no. 4, pp. 761-780, 2018.

[18] S. Boyd and L. Vandenberghe, Convex Optimization. Cambridge, U.K.: Cambridge Univ. Press, 2004.

[19] L. Paninski, "Estimation of entropy and mutual information," Neural Comput., vol. 15, no. 6, pp. 1191-1253, 2003.

[20] L. Paninski, "Asymptotic theory of information-theoretic experimental design," Neural Comput., vol. 17, no. 7, pp. 1480-1507, 2005.

[21] A. Meliou, A. Krause, C. Guestrin, and J. M. Hellerstein, "Nonmyopic informative path planning in spatio-temporal models," in Proc. 22nd Nat. Conf. Artif. Intell., 2007, pp. 602-607.

[22] W. C. Evans, D. Dias, S. Roelofsen, and A. Martinoli, "Environmental field estimation with hybrid-mobility sensor networks," in Proc. IEEE Int. Conf. Robot. Autom., 2016, pp. 5301-5308.

[23] K.-C. Ma, L. Liu, H. K. Heidarsson, and G. S. Sukhatme, "Data-driven learning and planning for environmental sampling," J. Field Robot., vol. 35, no. 5 , pp. 643-661, 2018

[24] A. Singh, A. Krause, C. Guestrin, and W. J. Kaiser, "Efficient informative sensing using multiple robots," J. Artif. Intell. Res., vol. 34, pp. 707-755, 2009.

[25] L. V. Nguyen, S. Kodagoda, and R. Ranasinghe, "Spatial sensor selection via Gaussian Markov random fields," IEEE Trans. Syst., Man, Cybern., Syst., vol. 46, no. 9, pp. 1226-1239, Sep. 2016.

[26] G. Hitz, E. Galceran, M.-E. Garneau, F. Pomerleau, and R. Siegwart, "Adaptive continuous-space informative path planning for online environmental monitoring," J. Field Robot., vol. 34, no. 8, pp. 1427-1449, 2017.
[27] M. L. Stein, Interpolation of Spatial Data: Some Theory for Kriging. New York, NY, USA: Springer-Verlag, 2012.

[28] C. Bishop, Pattern Recognition and Machine Learning. New York, NY, USA: Springer-Verlag, 2006.

[29] F. Lindgren and H. Rue, "Bayesian spatial modelling with R-INLA," $J$. Statist. Softw., vol. 63, no. 19, pp. 1-25, 2015.

[30] Intel Lab Data. [Online]. Available: http://db.csail.mit.edu/labdata/ labdata.html 